\title{
Broadband Liner Optimization for the Source Diagnostic Test Fan
}

\author{
Douglas M. Nark* and Michael G. Jones ${ }^{\dagger}$ \\ NASA Langley Research Center, Hampton, VA 23681-2199, U.S.A
}

\begin{abstract}
The broadband component of fan noise has grown in relevance with the utilization of increased bypass ratio and advanced fan designs. Thus, while the attenuation of fan tones remains paramount, the ability to simultaneously reduce broadband fan noise levels has become more appealing. This paper describes a broadband acoustic liner optimization study for the scale model Source Diagnostic Test fan. Specifically, in-duct attenuation predictions with a statistical fan source model are used to obtain optimum impedance spectra over a number of flow conditions for three liner locations in the bypass duct. The predicted optimum impedance information is then used with acoustic liner modeling tools to design liners aimed at producing impedance spectra that most closely match the predicted optimum values. Design selection is based on an acceptance criterion that provides the ability to apply increased weighting to specific frequencies and/or operating conditions. Typical tonal liner designs targeting single frequencies at one operating condition are first produced to provide baseline performance information. These are followed by multiple broadband design approaches culminating in a broadband liner targeting the full range of frequencies and operating conditions. The broadband liner is found to satisfy the optimum impedance objectives much better than the tonal liner designs. In addition, the broadband liner is found to provide better attenuation than the tonal designs over the full range of frequencies and operating conditions considered. Thus, the current study successfully establishes a process for the initial design and evaluation of novel broadband liner concepts for complex engine configurations.
\end{abstract}

\section{Introduction}

Accurate aircraft engine noise prediction is an important component of overall aircraft system noise assessment and the ability to predict fan noise within complex aircraft nacelle geometries is invaluable in optimizing acoustic liner treatment. With the utilization of increased bypass ratio and advanced fan designs, the broadband component of fan noise has grown in relevance. Thus, while the attenuation of fan tones remains paramount, the ability to simultaneously reduce broadband fan noise levels has become more attractive. In conjunction with this trend, a number of fan noise prediction assessments have provided valuable insights into improved fan noise propagation/radiation prediction with limited fan source information $\sqrt{1223}$ Advanced manufacturing techniques have also opened new possibilities for the practical implementation of broadband liner concepts ${ }^{4}$

With these observations in mind, a broadband acoustic liner optimization study for a benchmark scale model fan is pursued. Specifically, an acoustic duct propagation and radiation code is used to predict optimum impedance spectra over a number of flow conditions. Acoustic liner modeling tools are then used to identify geometric liner parameters (within manufacturing constraints) necessary to produce impedance spectra that most closely match the predicted optimum values. The resultant impedance values are then used with the propagation code to predict attenuation spectra and evaluate liner performance. While iteration between the liner design and evaluation stages is possible, the current study establishes a process for the initial design and evaluation of novel broadband liner concepts for complex engine configurations.

The remainder of the paper details this process and begins with the selection of the test configuration and operating conditions of interest in Section II A design metric for performance optimization is then defined and corresponding impedance spectra are generated. Section III] describes the specific computational approach, source specification, and optimum impedance prediction methodology used in this study. Specific modeling tools, candidate configurations, and acceptance criteria used in the subsequent liner modeling stage are presented in Section IV] Assessment of the various liner designs is then carried out to ensure acceptable performance, as discussed in Section $\mathrm{V}$. Finally, concluding remarks regarding some of the more significant results and further areas of interest are presented in Section VI

\footnotetext{
* Research Scientist, Structural Acoustics Branch, Research Directorate, AIAA Senior Member

$\dagger$ Senior Research Scientist, Structural Acoustics Branch, Research Directorate, AIAA Associate Fellow
} 


\section{Test Configuration}

For this study, the 22-inch diameter NASA/GE Source Diagnostic Test (SDT) far $\left[\frac{5677}{16}\right.$ chosen. The SDT fan, shown in Figure 11. has a design corrected tip speed of $1,215 \mathrm{ft} / \mathrm{sec}$, a stage pressure ratio of 1.47, and a bypass ratio of 8.9. The fan stage has 22 blades and 54 stator vanes in its baseline configuration. This configuration was chosen because of its use in previous studies $\frac{8|9| 10}{10}$ and the availability of measured acoustic dat 6 677 for further model validation. The cross-section of the SDT test configuration is shown in Figure 2 with candidate inlet and aft acoustic liner locations shaded in green. The three aft liner locations are the focus of this work and are assumed to have the same impedance. The fan speeds of interest, provided in Table 1, are representative of the three noise certification measurement points (approach, cutback, and takeoff). The mean flow associated with these fan speeds is based on Computational Fluid Dynamics (CFD) simulations of the aft bypass duct. From these simulations, the average Mach number at the trailing edge of the stator vanes, $M_{\text {ave }}(\mathrm{STE})$, is used as the starting value for one-dimensional Mach/Area calculations. The resultant Mach number distributions in the bypass duct are then used for in-duct propagation predictions. The frequencies considered are also included in Table 1 and are based on the blade passage frequency (BPF) and first harmonic (2BPF) at the approach, cutback, and takeoff fan speeds. This range of frequencies was selected to provide sufficient broadband coverage and including all six frequencies at each of the three flow speeds leads to a total of 18 different test points of interest. However at any particular flow condition, only the corresponding BPF and 2BPF frequencies represent likely tones, the other four frequencies represent broadband frequency points.

\section{Optimum Impedance Prediction}

A suitable code for performing the duct propagation and radiation predictions is the CDUCT-LaRC (CDL) code. This code calculates the propagation of a given acoustic source ahead of the fan face or aft of the exhaust guide vanes in the inlet or exhaust ducts, respectively. Subsequent to the propagation calculations, the code has the capability of computing the noise radiation field outside the duct. The three-dimensional duct may be acoustically lined (possibly circumferentially and radially segmented) in specified areas and incorporate struts/bifurcations. All of the modules that currently make up the CDL framework have been discussed previously ${ }^{11}$ and are utilized in this study. However, this discussion will focus on the propagation and radiation modules, as they are most pertinent to this investigation.

The duct propagation module is based on the CDUCT code developed by Dougherty ${ }^{12 \mid 13}$ and extended by Lan. 14 This code utilizes a parabolic approximation to the convected Helmholtz equation and offers a computationally efficient model that accounts for the complexities of fully three-dimensional nacelle configurations. The CDL code has been extended ${ }^{11}$ to support multi-block propagation calculations. The grid connectivity is determined and data is transferred from upstream to downstream blocks without user intervention. Results of the propagation module include the acoustic potential or pressure within the duct, which may be utilized by the radiation module for acoustic radiation calculations.

The duct radiation model is based on the Ffowcs Williams-Hawkings (FW-H) equation with a permeable data surface ${ }^{15}$ Use of this equation for many acoustic problems has shown it to generally provide better results than the Kirchhoff formula for moving surfaces. Based on the background flow conditions and propagation solution, this module calculates the radiated acoustic pressure at selected observer locations. Currently, the data surface is taken to be the nacelle inlet or exhaust plane for inlet or aft-fan cases, respectively. However, provisions have also been made to account for the effects of the shear layer on the radiation.

While CDL can accept arbitrary source specification (i.e., it is not a modal code), it is convenient to specify the acoustic source distribution in terms of duct modes. For situations in which the source pressure is available, this greatly simplifies the conversion to the required acoustic potential. However, when source information is not available, an assumption on the source description must be made. This is generally the case and the approach taken here follows that described by Zlavog and Eversman ${ }^{16 / 17}$ in a series of statistical studies into the effects of randomized modal source power and/or phase on attenuation in lined ducts. In this study, the source modal powers (and hence, amplitudes) and modal phases are allowed to vary randomly and independently. Thus, with equal probability of occurrence, the amplitude and phase for each cut-on source mode may take any value in the intervals from 0 to 1 and 0 to $2 \pi$, respectively. This uncertainty in source specification will undoubtedly result in uncertainty in the predicted sound levels. However, as discussed by Zlavog and Eversman,, 16 for the case of all propagating circumferential and radial acoustic modes with random modal power and phase, transmitted power appears to be normally distributed. In their statistical studies, this also produced statistical distributions with the least standard deviation. Therefore, as discussed in a previous CDL study, ${ }^{3}$ Student's t-distribution is used to statistically analyze the predicted attenuation results. For each configuration and power setting, 11 simulations are performed to produce a sample population from 
which mean, $\mu$, and standard deviation, $\sigma$, values can be inferred.

The optimal impedance spectra is predicted using the CDL code with the aforementioned source model. While a number of quantities may be used to formulate a cost function for the impedance optimization, the in-duct attenuation is chosen for this study. The impedance domain considered extends over a normalized resistance range of $0<\theta \leq 5$ and a normalized reactance range of $-5 \leq \chi \leq 5$. Initial step sizes of 0.5 are used to generate preliminary attenuation contours and optimum impedance prediction. A sub-domain with a step size of 0.2 is then generated about this impedance value to obtain a refined optimum impedance prediction. Thus, for a given frequency and flight condition, the optimum impedance is taken to be the value for which the predicted mean in-duct attenuation is maximum. It should be noted that while the in-duct attenuation is used to select the optimum impedance spectra, the acoustic radiation module provides the potential to study directivity patterns, which are relevant for comparison with test measurements and community noise assessment.

To further illustrate the optimum impedance prediction, the initial contours at the approach flow speed for the lowest (2863 Hz: BPF at approach) and highest (9282 Hz: 2BPF at takeoff) frequencies considered are shown in Figure 3 . Noting that the scales are the same in both plots, a much larger gradient can be seen about the maximum mean attenuation achieved at $2863 \mathrm{~Hz}$ versus that at $9282 \mathrm{~Hz}$. Thus, a much smaller sub-domain, extending beyond the initial domain boundary, is needed for the lower frequency. Recognizing the difficulty in matching the predicted optimum exactly, the contours are used to define a target "cloud" of impedance values in the neighborhood of optimum. In defining this target "cloud", one could simply accept any impedance value for which the predicted mean attenuation is within a certain tolerance (e.g., $2 \mathrm{~dB}$ ) of that achieved with the optimum impedance. However, the task of selecting an appropriate tolerance level remains. In this case, statistical information in the form of $95 \%$ confidence interval ${ }^{\mathrm{a}}$. which are different at each impedance value, is available. Therefore, the target "cloud" of impedance values is taken to include all impedances for which the $95 \%$ confidence interval overlaps that associated with the predicted optimum impedance value. Figure 4 illustrates this information for the mean attenuation results at $2863 \mathrm{~Hz}$. In Figure $4 \mathrm{a}$ contours indicate the "cloud" of impedance values described above for which the $95 \%$ confidence interval for the mean attenuation overlaps with that for the optimum impedance value. Figure $4 \mathrm{~b}$ shows the corresponding mean attenuation (indicated by the oval) at the optimum impedance with error bars representing the associated $95 \%$ confidence interval. For comparison purposes, the same information is provided in Figure 5 for $9282 \mathrm{~Hz}$ at the approach condition. At $2863 \mathrm{~Hz}$, it can be seen that the largest mean attenuation (with a relatively large 95\% confidence interval) is achieved over a relatively small impedance range. Conversely, at $9282 \mathrm{~Hz}$, a much lower mean attenuation (with a much smaller 95\% confidence interval) is achieved over a much larger range of impedance values.

Optimum impedance predictions for all six frequencies at the approach flow condition are consolidated in the normalized resistance and reactance plots in Figure 6 Optimum impedance values are denoted by circles. Error bars indicate the range of impedance values for which the $95 \%$ confidence interval overlaps that of the optimum impedance value. The dashed lines indicate the envelope of "acceptable" impedance values. Similar information was obtained for the cutback and takeoff conditions and is presented later.

\section{Liner Modeling}

As indicated above, the CDL propagation code is used to determine optimum impedance values for the liner locations in the aft bypass duct at selected flow conditions and frequencies. To achieve broadband performance, the liner was taken to be a multi-layer configuration by incorporating buried septa (or "mesh-caps") embedded into a honeycomb core. ${ }^{4}$ This concept allows the acoustic liner to be customized such that the surface impedance of each individual cell is independently controlled. This is achieved by the combination of parameters used to set the impedance in each cell. The various cells are then customized into a grid pattern of different mesh-cap depths or resistances within the acoustic panel to achieve a desired distributed impedance.

Clearly, geometric (e.g., liner geometric parameters such as porosity and core depth) and manufacturing (e.g., grid pattern size) constraints are key ingredients in this modeling phase, and must be taken into account to design acoustic liners that can be realistically achieved. In this study, the honeycomb cells are restricted to contain one meshcap. The mesh-cap depth, as well as its DC flow resistance, are allowed to vary from cell to cell. Finally, based on currently available manufacturing techniques, the customizable grid pattern was limited to a configuration of four cells. Representative liner configurations resulting from mesh-cap insertion are shown in Figure 7 to illustrate the two-layer setup and geometrical parameters.

All liners are assumed to have a 0.032 in $(0.081 \mathrm{~cm})$ thick perforate facesheet containing 0.032 in $(0.081 \mathrm{~cm})$

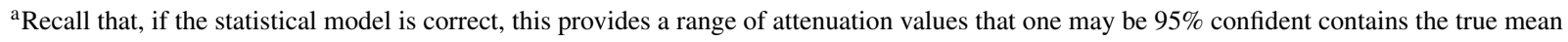
attenuation. 
diameter holes. Due to the relatively low target optimum resistance values, a rather unconventional $60 \%$ open area was selected for the facesheet. This minimized the impact of the facesheet on the overall resistance and allowed for greater flexibility in tuning the resistance of individual cells. The liner core is comprised of a 0.375 -inch $(0.953 \mathrm{~cm})$ diameter hexcel-shaped honeycomb core with an overall depth of 2.0 inches $(5.08 \mathrm{~cm})$. Mesh-cap materials ranging in DC flow resistance values from 600 to 1200 MKS Rayls were considered. Additionally, the distance between the mesh-cap and liner backplate, $h_{1}$, was allowed to vary (see Figure 7). In the design process, this value ranges from 0.20 in $(0.508 \mathrm{~cm})$ to 1.80 in $(4.57 \mathrm{~cm})$ in 0.20 in $(9.508 \mathrm{~cm})$ increments. An optimization process is used to determine the best combination of geometrical parameters to achieve the desired impedance values. Prediction tools based on the combination of a transmission line calculation ${ }^{18}$ and the Two-Parameter Impedance Prediction Mode ${ }^{19}$ are used to model the resultant liner impedance spectra.

Four design approaches are pursued using different combinations of the six frequencies and three flight conditions in Table 1 The CDL in-duct propagation results described in Section III]provide predicted optimum impedance values, $\zeta_{o p t, i}$, at each specific combination of flow speed and frequency. The liner modeling tools are then used to obtain design impedance values, $\zeta_{\text {pred }, i}$, that minimize the function

$$
F=\left\{\sum_{i=1}^{N} W_{i}\left(\zeta_{o p t, i}-\zeta_{p r e d, i}\right)\left(\zeta_{o p t, i}-\zeta_{\text {pred }, i}\right)^{*}\right\}^{0.5} .
$$

Here, the values $W_{i}$ are weighting values that may be used to assign increased importance to a selected number of frequencies and/or flow conditions and $N$ is the total number of frequency/flow condition combinations included. Note that with $W_{i}=1$ for all $N$, equation 1 reduces to the $L_{2}$ norm of the difference between the predicted optimum and the design impedance values. The initial approach focuses on a single frequency at the corresponding flow speed. For example, 2BPF at cutback (i.e., $8122 \mathrm{~Hz}$ ) is considered where $N=1$ and $W_{1}=1$ for that frequency and flow condition. These liner designs correspond to a typical approach where a single blade tone is targeted. Thus, six initial point designs (BPF and 2BPF at approach, cutback, and takeoff) were generated to provide a baseline for liner performance evaluation.

To illustrate these single tone designs, comparisons of optimum impedance values and those of the design targeting $2 \mathrm{BPF}$ at cutback are shown in Figures 8 - 10 In these figures, the design impedance values are denoted by squares and the envelope of "acceptable" impedance values is represented with dashed lines. Note that the impedance values at $8122 \mathrm{~Hz}$ are within the acceptance criteria across all three flow conditions. However, the impedance values (reactance in particular) at each of the other frequencies fall outside the impedance envelope for at least one operating condition. This is a typical characteristic of the baseline tonal liners.

Progressing toward a more broadband design, the second approach accounts for all six frequencies at one flow speed (e.g., cutback). This is followed by an approach that includes BPF and 2BPF at the corresponding flow conditions (i.e., $2863 \mathrm{~Hz}$ and $5726 \mathrm{~Hz}$ at the approach condition, $4061 \mathrm{~Hz}$ and 8122 at the cutback condition, etc.). In these two cases, $N=6$ and all weighting values are set to unity $\left(i . e ., W_{i}=1\right)$. Finally, all six frequencies at all flow speeds (approach, cutback, and takeoff) are considered using all of the predicted optimum impedance values $(N=18$ with $\left.W_{i}=1\right)$.

As a result of the constraint on the grid pattern size, the three aforementioned broadband approaches result in the same four cell configuration. Therefore, the resultant broadband liner design considering all frequencies and flow conditions $(N=18)$ actually incorporates the characteristics of the previous two approaches $(N=6)$. The impedance values for this liner design are presented in Figures 11-13. Again, the design impedance values are denoted by squares and the envelope of "acceptable" impedance values is represented with dashed lines. The broadband liner satisfies the optimum impedance objectives much better than the baseline liners. In fact, the impedance values at BPF and $2 \mathrm{BPF}$ for the cutback $(4061 \mathrm{~Hz}, 8122 \mathrm{~Hz})$ and takeoff $(4641 \mathrm{~Hz}, 9282 \mathrm{~Hz})$ conditions are within the acceptance criteria for all three flow conditions. It is at the approach fan speeds where difficulties may be identified. As discussed further in Section $\mathrm{V}$ it is the initial geometric constraints that make matching the optimum impedance values at this fan speed problematic.

\section{Liner Performance Evaluation}

The next step in the process is to use the design impedance values in the CDL code and evaluate the liner performance using in-duct attenuation. This is first performed for the baseline tonal liner designs. Representative results for the 2BPF/cutback tonal design are provided in Figure 14. In this figure, the mean attenuation achieved with the $2 \mathrm{BPF} /$ cutback tonal design is given by the dashed red line. For comparison purposes, the mean attenuation achieved 
with the optimum impedance values is given by the black solid line. Again, for both cases, the error bars represent the $95 \%$ confidence intervals for the mean attenuation. The liner performs well at the design frequency and its fundamental (see Figure 14b), as the 95\% confidence intervals overlap at these frequencies. However, the mean attenuation achieved at off-design frequencies is well below the optimum levels.

Alternatively, the mean attenuation achieved with the broadband liner is presented in Figure 15. In contrast to the $2 \mathrm{BPF} /$ cutback tonal liner, much better broadband performance is achieved without loss in attenuation at 2BPF/cutback. As might be expected from the comparison of impedance values, there is overlap in $95 \%$ confidence intervals at BPF and $2 \mathrm{BPF}$ for the cutback $(4061 \mathrm{~Hz}, 8122 \mathrm{~Hz})$ and takeoff $(4641 \mathrm{~Hz}, 9282 \mathrm{~Hz})$ conditions for all three flow conditions. However, the difficulties in matching impedance values at the approach fan speeds lead to poor performance at $2863 \mathrm{~Hz}$ and $5726 \mathrm{~Hz}$. As alluded to above, it is not possible to achieve the relatively low optimum resistance at $2863 \mathrm{~Hz}$ subject to the geometric constraints applied to the design. It is expected that this problem could be alleviated by allowing for the absence of a mesh-cap in at least one of the four cells. Although not pursued in the current study, this is a design modification to investigate in further iterations between the liner design and evaluation stages. Overall, the broadband design, even in its current iteration, offers superior performance over the baseline single frequency designs.

\section{Concluding Remarks}

A broadband acoustic liner optimization study was performed for the scale model SDT fan. Specifically, in-duct attenuation predictions are used to obtain optimum impedance spectra over a number of flow conditions for three liner locations in the bypass duct of the SDT. The predicted optimum resistance spectra show a general increase from the lowest frequency value that flattens with increasing frequency. This is accompanied by relatively flat reactance spectra. The source model selection also allows for the generation of $95 \%$ confidence intervals for the predicted mean attenuation. From this analysis, a general trend of lower mean attenuation accompanied by increasing larger envelopes of acceptable impedance values are also observed as frequency increases over all operating conditions.

The predicted optimum impedance information is used with acoustic liner modeling tools to design liner configurations aimed at producing impedance spectra that most closely match the predicted optimum values. Design selection is based on an acceptance criteria that provides the ability to apply increased weighting to specific frequencies and/or operating conditions. Typical tonal liner designs targeting single frequencies at one operating condition are first produced to provide baseline performance information. These are followed by multiple broadband design approaches that ultimately lead to a single broadband liner configuration. The broadband liner is found to satisfy the optimum impedance objectives much better than the tonal liner designs. In addition to the impedance spectra comparisons, the design impedance values are used in the propagation code to predict in-duct attenuation spectra for the purpose of quantitatively evaluating liner performance. As may be inferred from the impedance spectra, the broadband liner provides better attenuation than the tonal designs over the full range of frequencies and operating conditions.

Through the course of this study, a number of opportunities for further research have also been identified. First, the selection of the SDT fan affords the opportunity to study the effects of the acoustic source model on the final liner design. As mentioned above, previous studies yielded measured fan source information that may be used to check specific design points. Additionally, the three aft liner locations may be treated separately so that the performance of a variable liner topology may be investigated. Regarding liner design, the initial geometric constraints led to some difficulty in matching the optimum impedance at the lowest frequency. Therefore, while not pursued in this study, further iteration between the liner design and evaluation stages would be of interest for improving the broadband design. Finally, to extend the liner performance assessment, a metric based on the integrated attenuation spectra (on a power basis) over the full frequency range may be employed. As with the design impedance cost function (Eq. (1), weighting of specific frequencies/operating conditions could also be introduced in this performance metric.

In addition to these areas of interest, this work has provided an excellent opportunity to perform an integrated study of duct acoustic propagation/radiation and liner modeling tools. A foundation is set for future comparison with other duct propagation/liner modeling tools and a process for the initial design and evaluation of novel broadband liner concepts for complex engine configurations has been successfully developed.

\section{Acknowledgments}

The authors wish to thank Dr. Dan Tweedt and Dr. Chris Hughes for their assistance with SDT geometry and flow conditions. This research was funded by the Subsonic Fixed Wing Project of NASA's Fundamental Aeronautics Program. 


\section{References}

${ }^{1}$ Schuster, B., Lieber, L., and Vavalle, A., "Optimziation of a Seamless Inlet Liner Using an Empirically Validated Prediction Method," AIAA Paper 2010-3824, 2010.

${ }^{2}$ Weir, D., "Engine Validation of Noise and Emission Reduction Technology Phase I," NASA CR 2008-215225, 2008.

${ }^{3}$ Nark, D. M., "Assessment of Radiated Fan Noise Prediction Capabilities using Static Engine Test Data," AIAA Paper 2011-2807, 2011.

${ }^{4}$ Jones, M. G., Howerton, B. M., and Ayle, E., "Evaluation of Parallel-Element, Variable-Impedance, Broadband Acoustic Liner Concepts," 18th AIAA/CEAS Aeroacoustics Conference, American Institute of Aeronautics and Astronautics, Reston, VA (submitted for publication).

${ }^{5}$ Hughes, C. E., “Aerodynamic Performance of Scale-Model Turbofan Outlet Guide Vanes Designed for Low Noise," AIAA Paper 2002-0374, 2002.

${ }^{6}$ Heidelberg, L. J., "Fan Noise Source Diagnostic Test - Tone Modal Structure Results,” AIAA Paper 2002-2428, 2002.

${ }^{7}$ Woodward, R. P., Hughes, C. E., Jeracki, R. J., and Miller, C. J., "Fan Noise Source Diagnostic Test - Far Field Acoustic Results," AIAA Paper 2002-2427, 2002.

${ }^{8}$ Nark, D. M., Envia, E., and Burley, C. L., "On Acoustic Source Specification for Rotor-Stator Interaction Noise Prediction,” AIAA Paper 2010-3713, 2010.

${ }^{9}$ Nark, D. M., Envia, E., and Burley, C. L., "Fan Noise Prediction with Applications to Aircraft System Noise Assessment," AIAA Paper 2009-3291, 2009.

${ }^{10}$ Envia, E., Tweedt, D. L., Wooward, R. P., Elliott, D. M., Fite, E. B., Hughes, C. E., Podboy, G. G., and Sutliff, D. L., "An Assessment of Current Fan Noise Prediction Capability," AIAA Paper 2008-2991, 2008.

${ }^{11}$ Nark, D. M., Farassat, F., Pope, D. S., and Vatsa, V., "The Development of the Ducted Fan Noise Propagation and Radiation Code CDUCTLaRC," AIAA Paper 2003-3242, 2003.

${ }^{12}$ Dougherty, R. P., “A Wave-Splitting Technique for Nacelle Acoustic Propagation,” AIAA Paper 97-1652, 1997.

${ }^{13}$ Dougherty, R. P., "A Parabolic Approximation for Flow Effects on Sound Propagation in Nonuniform, Softwall, Ducts," AIAA Paper 99$1822,1999$.

${ }^{14}$ Lan, J. H., “Turbofan Duct Propagation Model,” NASA CR 2001-211245, 2001.

${ }^{15}$ Brentner, K. B. and Farassat, F., "Analytical Comparison of the Acoustic Analogy and Kirchhoff Formulation for Moving Surfaces," AIAA Journal, Vol. 36, No. 8, 1998, pp. 1379-1386.

${ }^{16}$ Zlavog, G. and Eversman, W., "Source effects on attenuation in lined ducts. Part I: A statistically based computational approach," Journal of Sound and Vibration, Vol. 307, No. 1-2, 2007, pp. 113-138.

${ }^{17}$ Zlavog, G. and Eversman, W., "Source effects on attenuation in lined ducts. Part II: Statistical properties," Journal of Sound and Vibration, Vol. 307, No. 1-2, 2007, pp. 139-151.

${ }^{18}$ Parrott, T. L. and Jones, M. G., "Parallel-Element Liner Impedances for Improved Absorption of Broadband Sound in Ducts," Noise Control Engineering Journal, Vol. 43, No. 6, 1995, pp. 183-195.

${ }^{19}$ Jones, M. G., Parrott, T. L., and Watson, W. R., "Uncertainty and Sensitivity Analyses of a Two-Parameter Impedance Prediction Model," AIAA Paper 2008-2928, 2008.

\begin{tabular}{|c|c|c|c|c|c|}
\hline Condition & $M_{\text {ave }}(\mathrm{STE})$ & Corrected RPM & \% Design Speed & BPF $(\mathrm{Hz})$ & 2BPF $(\mathrm{Hz})$ \\
\hline \hline Approach & 0.262 & 7,809 & 61.7 & 2863 & 5726 \\
\hline Cutback & 0.343 & 11,075 & 87.5 & 4061 & 8122 \\
\hline Takeoff & 0.374 & 12,657 & 100.0 & 4641 & 9282 \\
\hline
\end{tabular}

Table 1: SDT Model Fan Tip Speed Conditions 


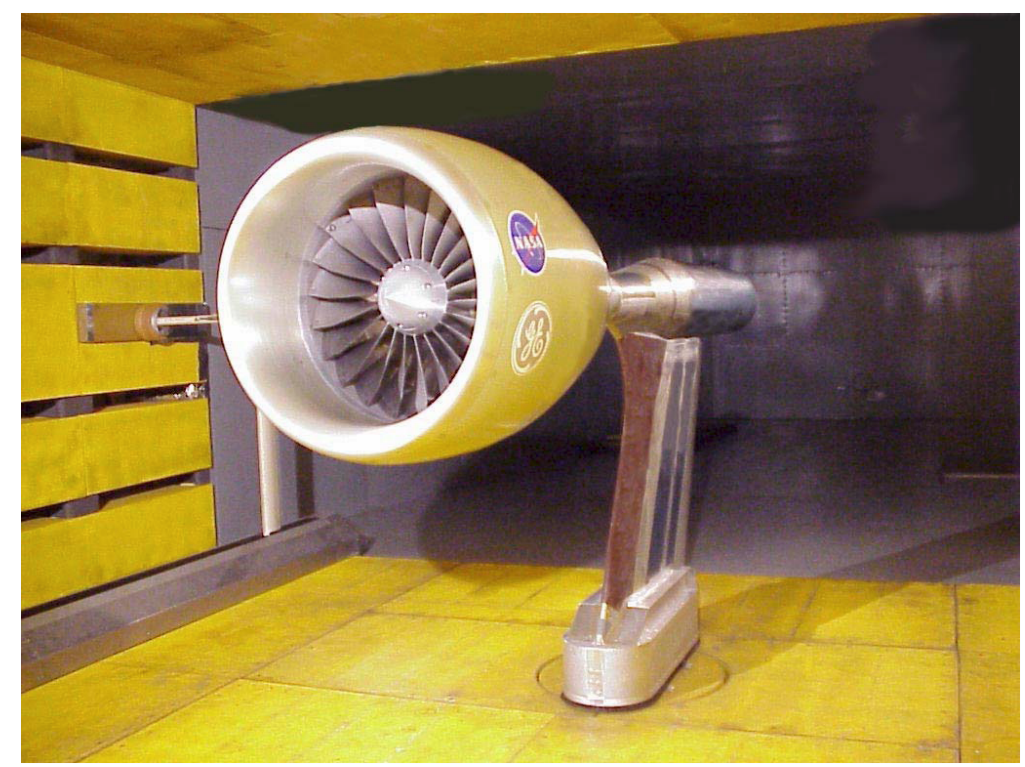

Figure 1: Source Diagnostic Test (SDT) model fan installed in the 9'x15' Low Speed Wind Tunnel.

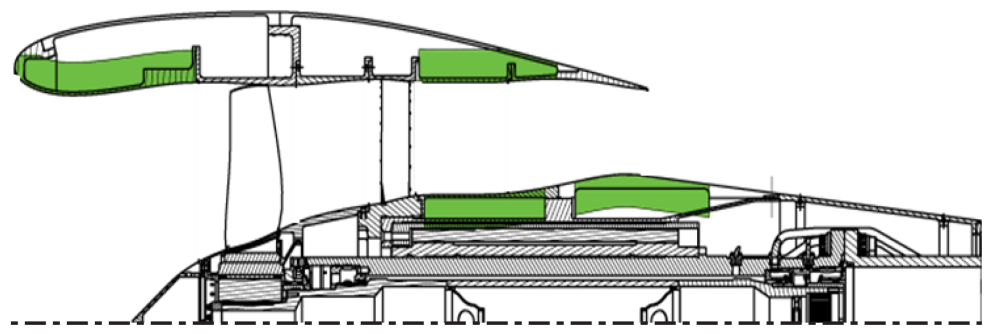

Figure 2: SDT model fan stage in baseline configuration with acoustic liner locations denoted in green.

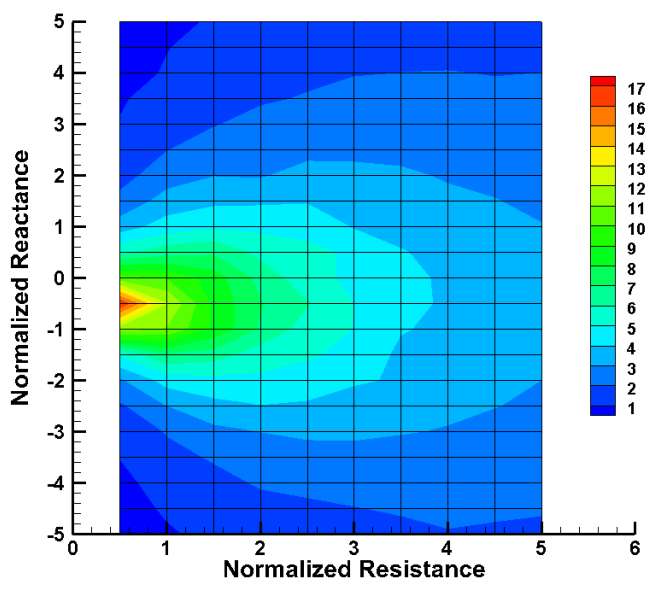

(a) Approach BPF $(2863 \mathrm{~Hz})$

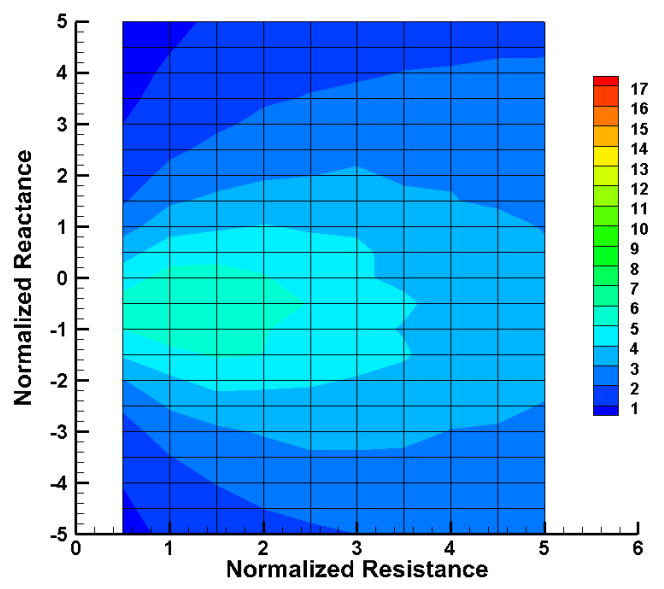

(b) Takeoff 2BPF (9282 Hz)

Figure 3: Mean attenuation contours (Approach flow condition). 


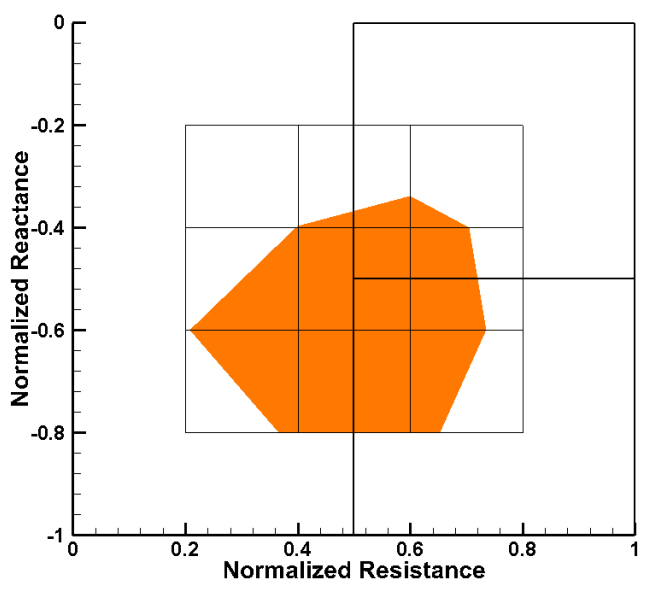

(a) Optimum Impedance

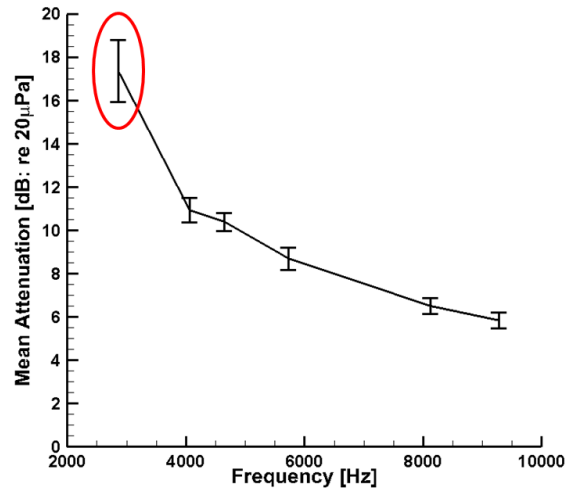

(b) Optimum Attenuation

Figure 4: Mean attenuation contours and associated confidence intervals (Approach BPF: $2863 \mathrm{~Hz}$ ). Contours in Figure $4 a$ represent impedance values for which the $95 \%$ confidence interval for the mean attenuation overlaps with that for the optimum impedance value. Errors bars in Figure $4 b$ represent the 95\% confidence interval in the mean attenuation at the optimum impedance.

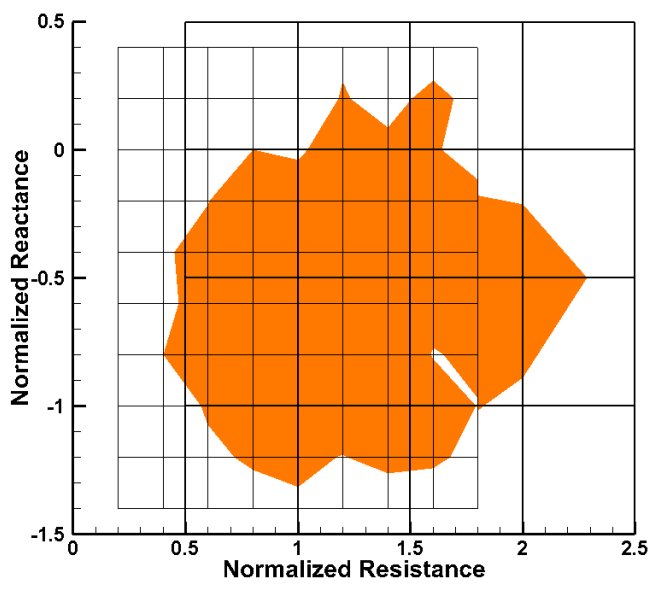

(a) Optimum Impedance

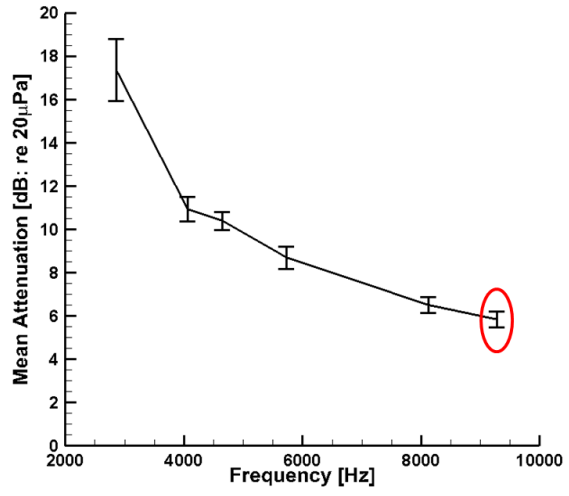

(b) Optimum Attenuation

Figure 5: Mean attenuation contours and associated confidence intervals (Takeoff 2BPF: $9282 \mathrm{~Hz}$ ). Contours in Figure 5a represent impedance values for which the $95 \%$ confidence interval for the mean attenuation overlaps with that for the optimum impedance value. Errors bars in Figure 5 b represent the $95 \%$ confidence interval in the mean attenuation at the optimum impedance. 


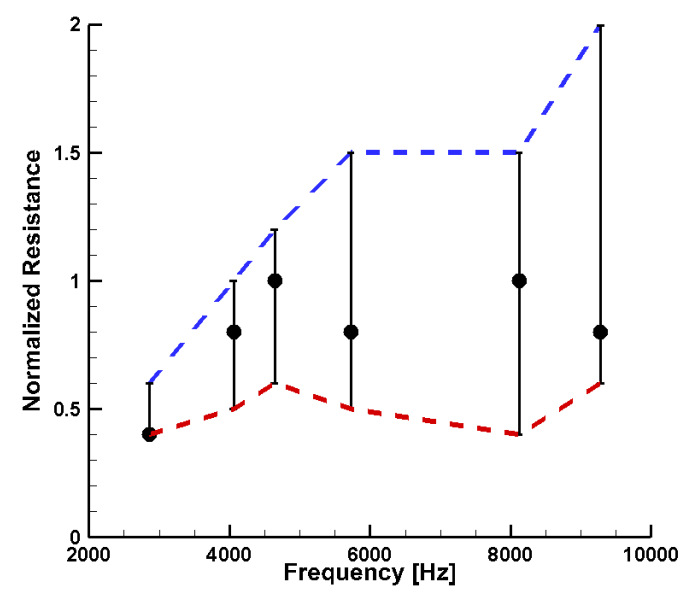

(a) Normalized Resistance

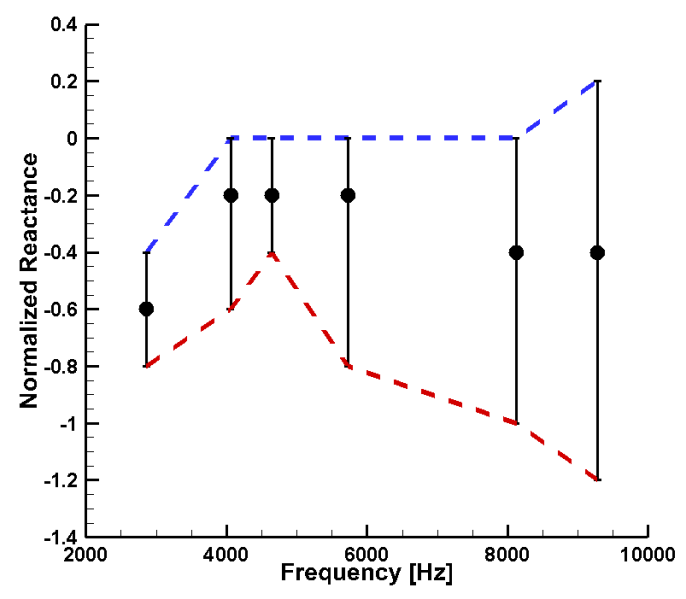

(b) Normalized Reactance

Figure 6: Optimum impedance values denoted by circles (Approach flow condition). Error bars indicate impedance values for which the $95 \%$ confidence interval for the mean attenuation overlaps with that for the optimum impedance value.

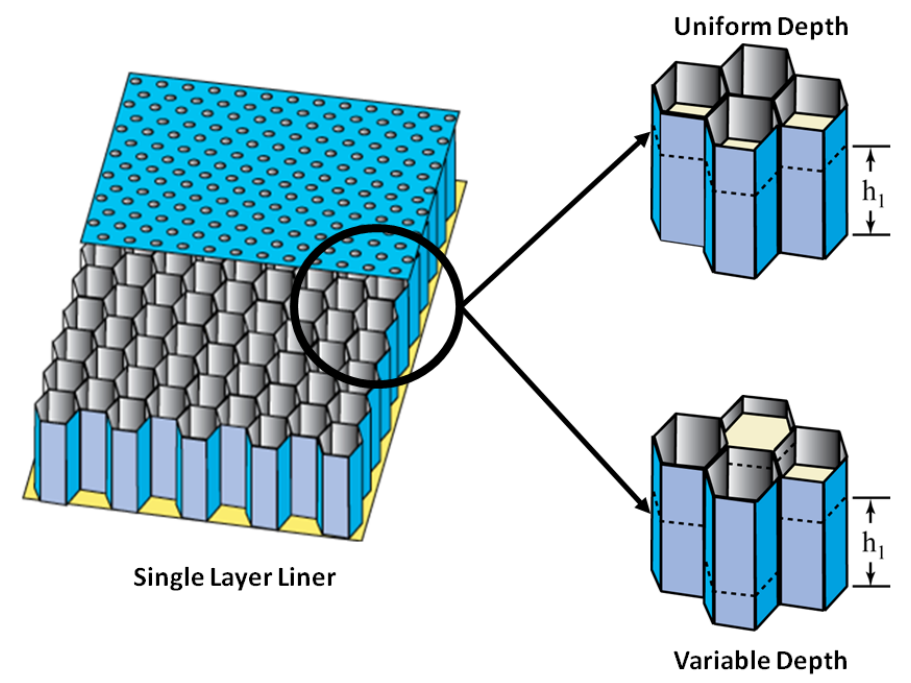

Figure 7: Parent single layer liner and resultant two-layer liner configurations (uniform and variable depth) via meshcap insertion. 


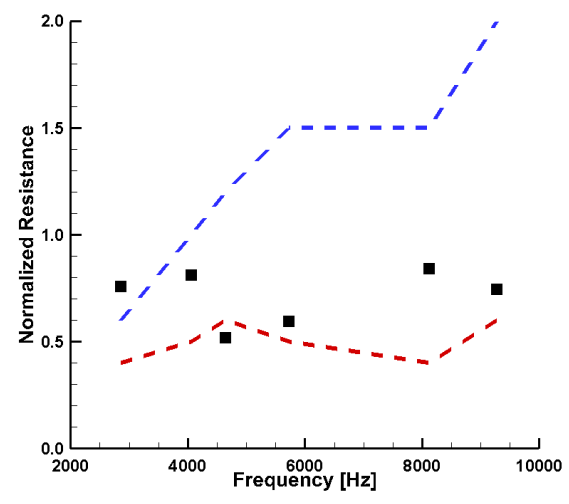

(a) Normalized Resistance

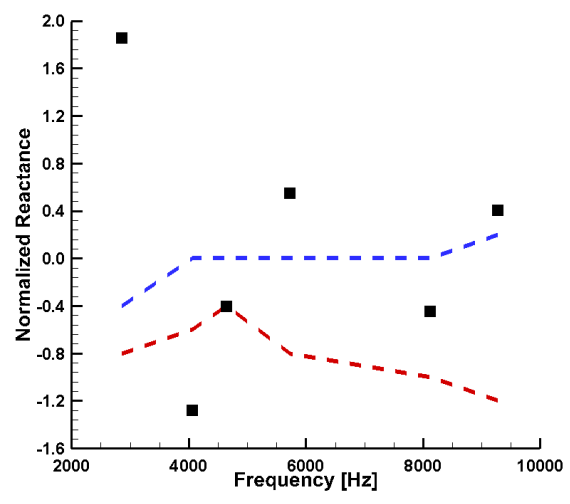

(b) Normalized Reactance

Figure 8: Cutback 2BPF (8122 Hz) design impedance values denoted by squares (Approach flow condition).

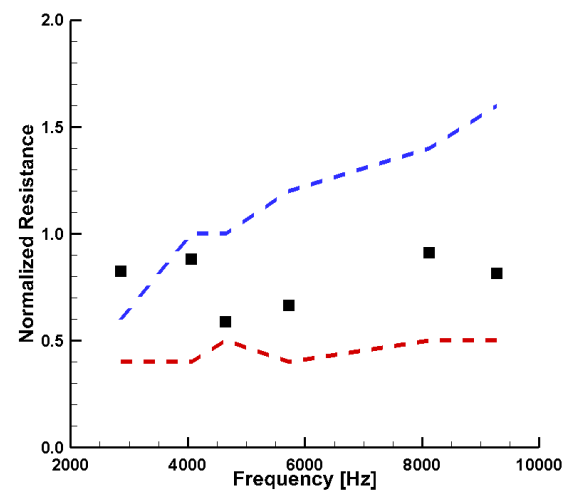

(a) Normalized Resistance

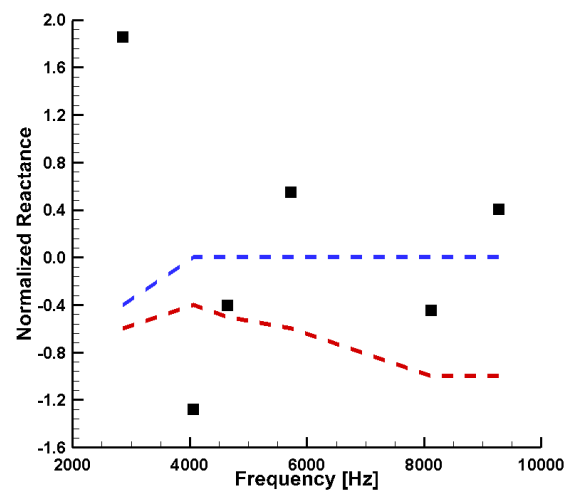

(b) Normalized Reactance

Figure 9: Cutback 2BPF ( $8122 \mathrm{~Hz})$ design impedance values denoted by squares (Cutback flow condition).

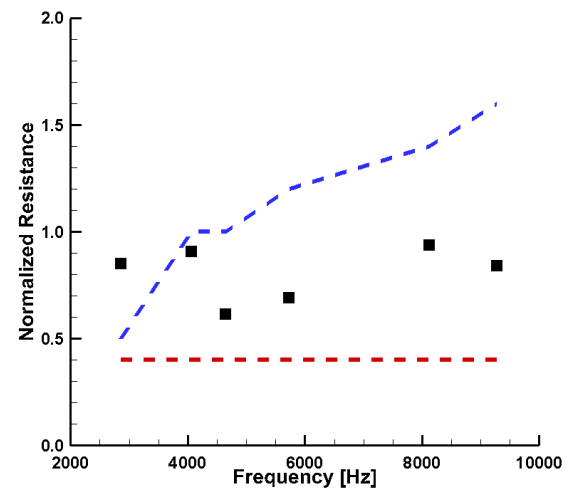

(a) Normalized Resistance

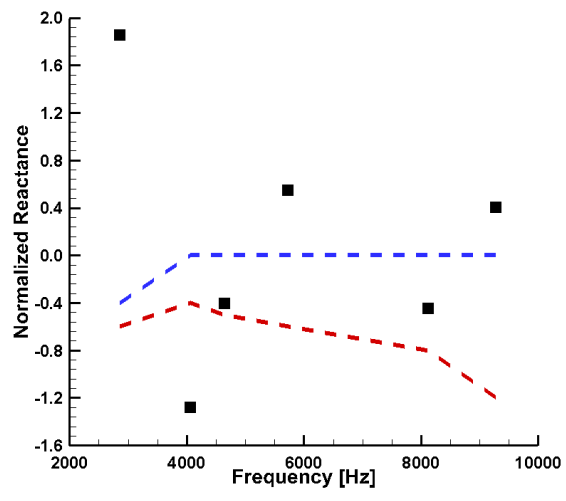

(b) Normalized Reactance

Figure 10: Cutback 2BPF ( $8122 \mathrm{~Hz}$ ) design impedance values denoted by squares (Takeoff flow condition). 


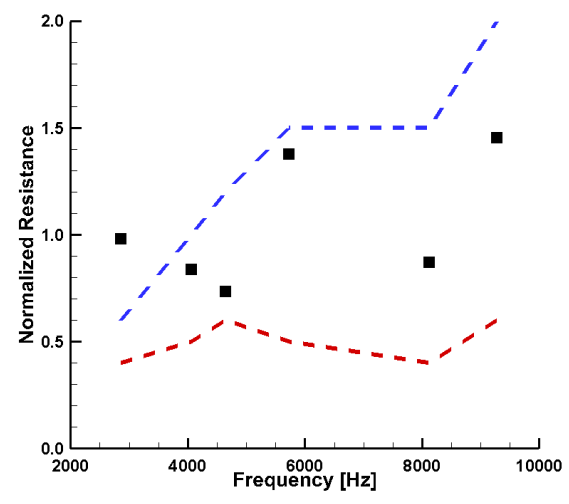

(a) Normalized Resistance

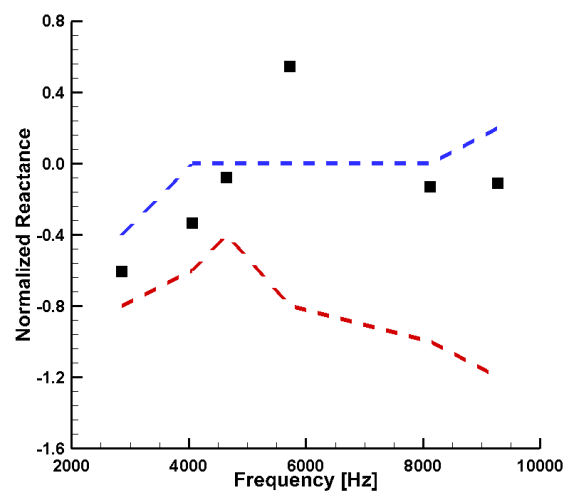

(b) Normalized Reactance

Figure 11: Broadband design impedance values denoted by squares (Approach flow condition).

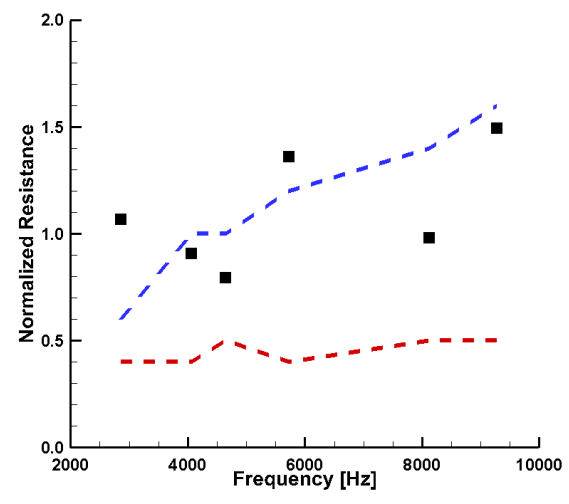

(a) Normalized Resistance

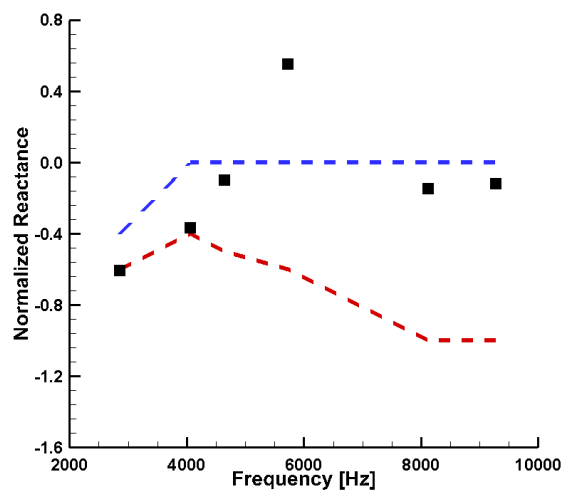

(b) Normalized Reactance

Figure 12: Broadband design impedance values denoted by squares (Cutback flow condition).

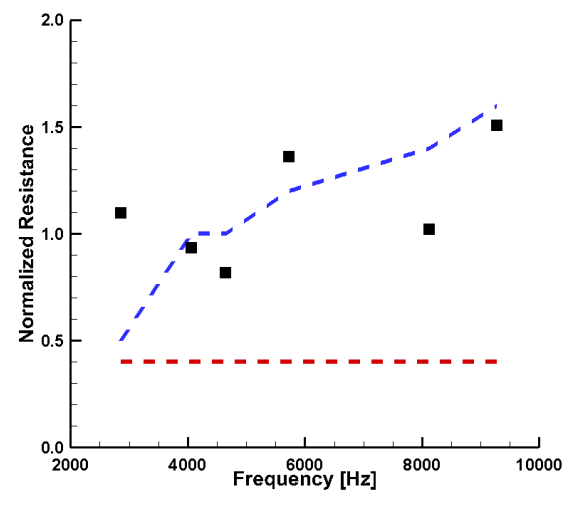

(a) Normalized Resistance

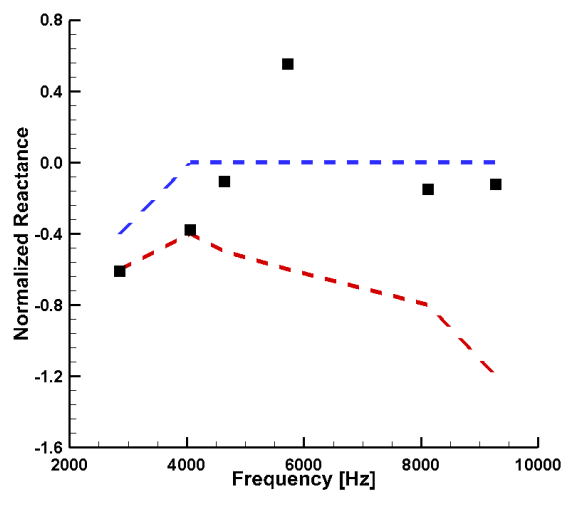

(b) Normalized Reactance

Figure 13: Broadband design impedance values denoted by squares (Takeoff flow condition). 


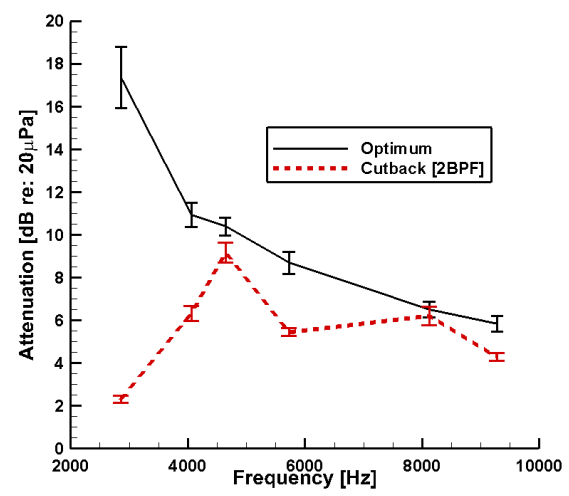

(a) Approach flow condition

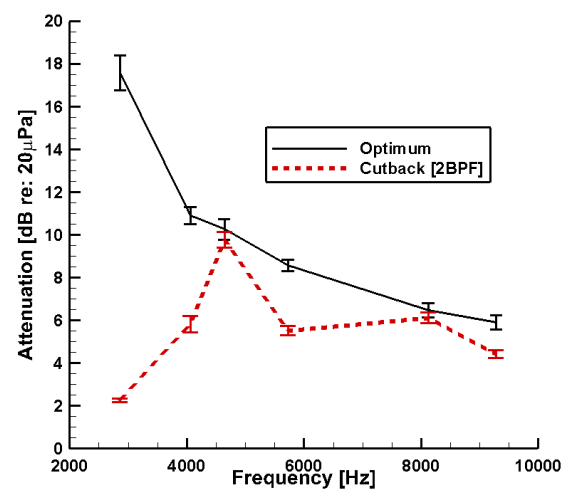

(b) Cutback flow condition

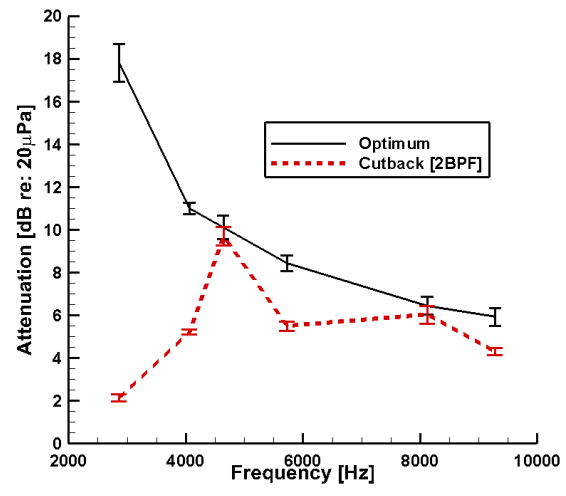

(c) Takeoff flow condition

Figure 14: Cutback 2BPF design attenuation values. Error bars indicate $95 \%$ confidence intervals. 


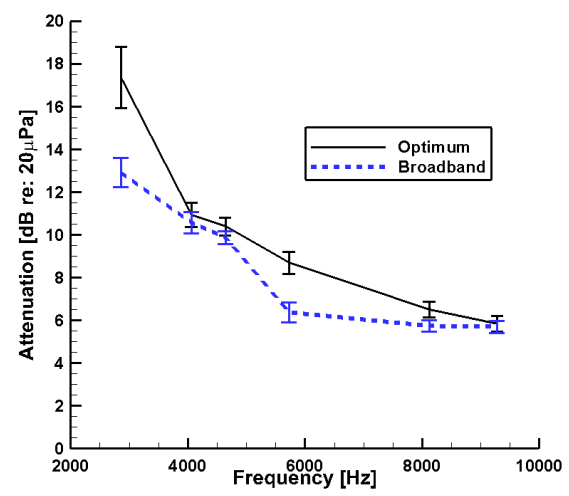

(a) Approach flow condition

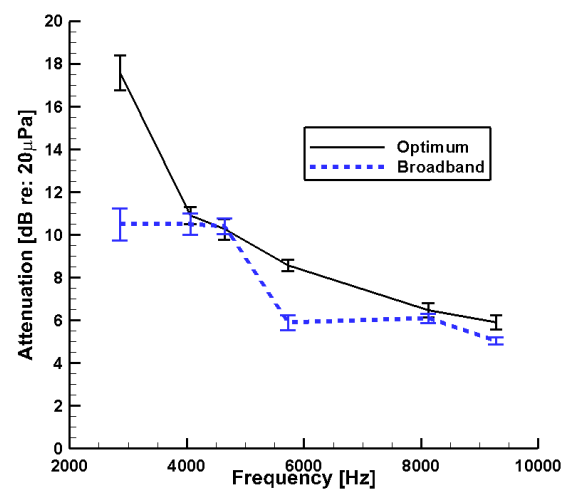

(b) Cutback flow condition

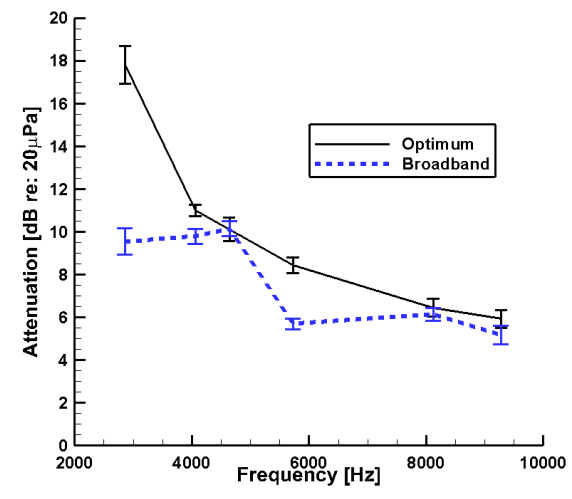

(c) Takeoff flow condition

Figure 15: Broadband design attenuation values. Error bars indicate 95\% confidence intervals. 